\title{
Long-term observation of adults after successful repair of aortic coarctation
}

\author{
Beata Róg1, Magdalena Okólska ${ }^{1}$ Piotr Weryński², Piotr Wilkołek³, Tomasz Pawelec ${ }^{4}$, Jacek Pająk \\ Piotr Podolec ${ }^{3}$, Lidia Tomkiewicz-Pająk ${ }^{3}$ \\ ${ }^{1}$ Cardiological Outpatient Clinic, Department of Cardiovascular Diseases, John Paul II Hospital, Krakow, Poland \\ ${ }^{2}$ Department of Pediatric Cardiology, Jagiellonian University Medical College, Krakow, Poland \\ ${ }^{3}$ Department of Cardiac and Vascular Diseases, Institute of Cardiology, Jagiellonian University Medical College, John Paul II Hospital, \\ Krakow, Poland \\ ${ }^{4}$ Department of Interventional Cardiology, Jagiellonian University Medical College and John Paul II Hospital, Krakow, Poland \\ ${ }^{5}$ Pediatric Heart Surgery Department and General Pediatric Surgery Department, Medical University of Warsaw, Warsaw, Poland
}

Adv Interv Cardiol 2019; 15, 4 (58): 455-464 DOI: https://doi.org/10.5114/aic.2019.90220

\begin{abstract}
A bstract
Introduction: Despite successful repair of aortic coarctation, cardiovascular complications occur.

Aim: To analyse type and frequency of late complications and their impact on exercise capacity in adults after aortic coarctation repair. Material and methods: Fifty-eight adults after aortic coarctation repair, 36 male, median age $27.46 \pm 10.57$, were compared to 30 healthy volunteers. Physical examination, transthoracic echocardiography, carotid intima-media thickness measurement, cardiopulmonary exercise test and 24-hour ambulatory blood pressure monitoring were performed.

Results: The main complications were: arterial hypertension $48.3 \%$, myocardial hypertrophy in echocardiography $29.34 \%$, recoarctation $25.86 \%$, aortic dilation $13.79 \%$ and coronary artery disease $6.89 \%$. Exercise tolerance was reduced in the cardiopulmonary exercise test. The $\mathrm{VO}_{2} / \mathrm{kg}$ peak was lower, $29.01 \pm 8.79$ vs. $49.16 \pm 7.38 \mathrm{ml} / \mathrm{kg} / \mathrm{min}, p<0.001$, VE $/ \mathrm{VCO}$, peak higher $28.18 \pm 4.69$ vs. $26.78 \pm 3.13, p=0.017$. The peak heart rate was reduced, $157.28 \pm 22.22$ vs. $177.93 \pm 23.08 \mathrm{bpm}, p<0.001$, peak systolic blood pressure was higher, $174.79 \pm 17.62$ vs. $153.33 \pm 4.79 \mathrm{~mm} \mathrm{Hg}, p<0.001$. Systolic blood pressure in 24-hour ambulatory monitoring correlated with left ventricle mass index, $r=0.29, p=0.025$, wall thickness, $r=0.31, p=0.039$. Age at operation was related to left ventricle wall thickness, $r=0.27, p=0.041$, and carotid intima-media thickness, $r=0.26, p=0.046$. There was no association of any cardio-pulmonary parameters with time from surgery, type of operation or echocardiography results.

Conclusions: Adults after aortic coarctation repair suffer from arterial hypertension, recurrent aortic stenosis, aortic aneurysms, and coronary artery disease. Reduced exercise capacity in cardio-pulmonary exercise test is related to hypertensive reaction and chronotropic incompetence.
\end{abstract}

Key words: atherosclerosis risk factors, coarctation of aorta, exercise capacity, cardio-pulmonary exercise test, long-term complications in coarctation of the aorta.

S u m m a ry

Despite successful repair of aortic coarctation, cardiovascular complications occur. The aim of this study was to analyse type and frequency of late complications and their impact on exercise capacity in adults after aortic coarctation repair. Adults after aortic coarctation repair suffer from arterial hypertension, recurrent aortic stenosis, aortic aneurysms, and coronary artery disease. Reduced exercise capacity in the cardio-pulmonary exercise test is related to hypertensive reaction and chronotropic incompetence.

\section{Introduction}

Coarctation of the aorta (COA) is a congenital narrowing of the descending aorta at the level of the subclavian artery [1]. It is the fifth most common congenital heart disorder with a prevalence from $5 \%$ to $9 \%$ of all congenital heart diseases [2] and 2:1 predominance in males [3].

\section{Corresponding author:}

Lidia Tomkiewicz-Pająk MD, PhD, Department of Cardiac and Vascular Diseases, Institute of Cardiology, John Paul II Hospital, 80 Prądnicka St, 31-202 Krakow, Poland, e-mail: Itom@wp.pl

Received: 5.06.2019, accepted: 17.09.2019. 
Aortic coarctation may occur as a discrete stenosis; it can also be related to long segment narrowing, hypoplasia of the aortic arch, and stenosis of the abdominal aorta, and may have collateral vessels [4]. Unrepaired CoA is associated with congestive heart failure, aortic rupture, infectious endocarditis and intracranial haemorrhage [2] and mortality of more than $80 \%$ by the age 50 years [3]. Currently, several treatment options are available, including surgical and transcatheter interventions by balloon angioplasty and stenting, with procedure mortality $<1 \%$ [1]. Despite improving short-term results, survival after aortic coarctation repair is decreased, with a survival rate of $74 \%$ at 30 years of follow-up [1], due to increased arterial stiffness, arterial hypertension, ischaemic heart disease, atherosclerosis, chronic heart failure, aortic root aneurysms and cerebral vascular accidents [5-10]. Despite a successful repair of aortic coarctation, which improves prognosis and prolongs life, cardiovascular complications occur in most convalescents: arterial hypertension in $32.5 \%$ of CoA patients [11], recoarctation of the aorta in $3-26 \%$ [5], ischaemic heart disease in 4.9\% [12], aortic aneurysms in $26 \%$ [5].

\section{Aim}

The aim of this study was to describe and analyze the type and frequency of late complications in adult patients after coarctation of the aorta repair, and to assess their impact on exercise capacity.

\section{Material and methods}

Fifty-eight adult patients after coarctation of aorta repair being under control of the Cardiology Institute in Krakow, Poland were included in the study. They were compared to 30 healthy, age- and sex-matched volunteers. All the participants underwent physical examination, ambulatory blood pressure measurement, transthoracic echocardiography, carotid intima-media thickness estimation and cardio-pulmonary exercise test. The clinical analysis included: age, body mass index (BMI), body surface area (BSA), arm and leg blood pressure measurement. Data about age at surgery, type of operation, occurrence of recoarctation, vascular complications (e.g. arterial hypertension, coronary artery disease, stroke) and therapy were collected. The patients were included in the study if they were aged $\geq 18$ years old and clinically stable for at least 3 months before examination. The exclusion criteria were: acute vascular or inflammatory illness, pregnancy, neoplastic disease, physical disability making cardiopulmonary exercise test unfeasible (one person was excluded due to paraplegia caused by spinal cord damage after operation) and mental disorder making it impossible to obtain informed consent. Patients with coexisting severe congenital heart diseases (e.g. single ventricle heart, transposition of the great arteries) were excluded to avoid their influence on exercise capacity in the cardiopulmonary test. All participants presented in the echocardiography preserved systolic function of the left ventricle (ejection fraction above $50 \%$ by Simpson estimation). None of the study group presented an armleg pressure gradient above $20 \mathrm{~mm} \mathrm{Hg}$. In the case of restenosis in medical history, patients underwent cardiac catheterization before this research and were treated with percutaneous angioplasty or stent implantation with a successful result in hemodynamic measurement.

All the participants signed informed consent before enrolling in the study. The study protocol followed the Helsinki Declaration and was approved by the local ethical committee (license no. 122.6120.27.2016).

\section{4-hour ambulatory blood pressure measurement (ABPM)}

Twenty-four hour analysis of the arterial blood pressure was conducted using an automatic sphygmomanometer (automatic sphygmomanometr (Spacelabs Healthcare)). The measurement was taken at the right arm every 15 min during the day and every 30 min during the night (daytime 7 a.m. to 10 p.m., night-time 10 p.m. to 7 a.m.). The average values of the systolic and diastolic pressure were calculated from all scores; the recordings were considered when they included more than $80 \%$ valid measurements. The anti hypertensive therapy (if indicated previously) was continued during the examination. Values higher than $135 / 85 \mathrm{~mm} \mathrm{Hg}$ during the daytime and above $120 / 70 \mathrm{~mm} \mathrm{Hg}$ at night-time were considered indicative of arterial hypertension [13].

\section{Transthoracic echocardiography (TTE)}

All subjects underwent comprehensive transthoracic echocardiography using the Vivid 7 system, General Electric Medical Systems, USA with a $2.5 \mathrm{MHz}$ probe in 2D, $M$ and Doppler modes. The left ventricle systolic (Biplane Simpson method) and diastolic function (Tissue Doppler Imaging) were estimated as well as the left ventricle wall thickness and mass, which was indexed for body surface area. The concomitant valvular heart diseases were acquired. The aortic root diameter and a peak systolic transisthmic gradient were obtained from the suprasternal window. According to ESC guidelines [13] left ventricular hypertrophy was diagnosed if left ventricular mass index (LVMI) was higher than $115 \mathrm{~g} / \mathrm{m}^{2}$ for men and $95 \mathrm{~g} / \mathrm{m}^{2}$ for women, while impaired systolic left ventricle function was diagnosed when ejection fraction measured by the Simpson method extended below $50 \%$.

\section{Carotid intima-media thickness (CIMT)}

The measurements were obtained from the arterial wall segments of the right and left common carotid arteries, carotid bulbs and internal carotid arteries. The mean CIMT was defined as the mean CIMT of the 
near and far walls from both the left and right carotid arteries from three measurements [14]. The Vivid 7 system, General Electric Medical Systems, USA with 7.5 to $10 \mathrm{MHz}$ linear probe was used. The examinations were conducted by one experienced physician, with no blinded results.

\section{Cardiopulmonary exercise test (CPET)}

The cardiopulmonary exercise test was performed to evaluate the exercise tolerance in the symptom limited modified Bruce protocol. Oxygen uptake $\left(\mathrm{VO}_{2}\right)$, carbon dioxide production $\left(\mathrm{VCO}_{2}\right)$ and minute ventilation (VE) were measured at rest and peak exercise with a computerized analyser. The peak oxygen uptake $\mathrm{VO}_{2} /$ $\mathrm{kg}$ peak $(\mathrm{ml} / \mathrm{kg} / \mathrm{min})$ determined by the highest value of workload, peak heart rate and percentage of maximal heart rate were assessed. The ventilatory anaerobic threshold was calculated by means of the V-slope method. The ventilatory equivalent for carbon dioxide $\left(\mathrm{VE} / \mathrm{VCO}_{2}\right)$ was calculated as the amount of ventilation needed for the elimination of a given amount of carbon dioxide. The respiratory exchange ratio (RER) was assessed by dividing $\mathrm{VCO}_{2}$ by $\mathrm{VO}_{2}[15,16]$. The cardiopulmonary exercise test was considered as maximal if the respiratory quotient (carbon dioxide production divided by oxygen consumption) was higher than 1.1 , maximal heart rate higher than $85 \%$ of the age predicted maximal heart rate or maximal exertion of the patient occurred. Exercise capacity was quantified as metabolic equivalents (METs); one metabolic equivalent is the amount of oxygen consumed at rest and is equivalent to $3.5 \mathrm{ml} \mathrm{O} / \mathrm{kg}$ body $\times$ min. The blood pressure was measured at rest, every stage during the exercise, at peak workload and at recovery. All the anti-hypertensive therapy (including $\beta$-blockers) was suspended $48 \mathrm{~h}$ before the exercise test.

\section{Statistical analysis}

The continuous data were presented by means of the average value with the standard deviation or by median with the lower and upper quartile in the case of non-normal distribution of the data. To compare the averages between three different populations one-way ANOVA was performed if all assumptions were met, otherwise the Kruskal-Wallis test or Welch test was used. In the case of significant results of one of those analyses, a post-hoc test was applied. Normality was verified by means of the Shapiro-Wilk test, and the Levene test was applied to investigate heterogeneity of variance. Correlation between two continuous variables was assessed based on the Pearson or Spearman coefficient. Results with a $p$-value lower than the significance level $\alpha=0.05$ were considered as statistically significant. The calculations were performed using the R statistical package version 3.3.1 (www.r-project.org).

\section{Results}

Fifty-eight adult patients after coarctation of aorta repair were included in the study, 36 male, 22 female, median age $27.46 \pm 10.57$ years. They were compared to 30 healthy, age- and sex-matched volunteers. The median age at coarctation operation time was $8.68 \pm 8.64$ years, the median follow-up time was $20.39 \pm 9.8$ years. The most common type of operation was Dacron/Gore-tex patch repair in 25 (43.1\%) patients, 18 (31.03\%) were operated on by Waldhausen subclavian flap angioplasty, 7 (12.06\%) by end-to-end anastomosis and 8 (13.8\%) underwent percutaneous angioplasty with stent implantation in adolescent or adult age. Fifteen (25.86\%) were operated on due to concomitant ventricle septal defect (VSD), 6 (12.07\%) due to persistent ductus arteriosus (PDA), 10 (17.24\%) because of hypoplastic aortic arch, 1 (1.72\%) person underwent aortic valve replacement (AVR) and 4 (6.89\%) Bentall de Bono operation due to aortic valve dysfunction and aortic aneurysm. In the CoA group, there were 36 (62.06\%) people with bicuspid aortic valve. Patients with recoarctation $(15,25.86 \%)$ in medical history were treated at median age $15.5 \pm 8.17$ years. None of the observed patients had a blood pressure (BP) difference $>20 \mathrm{~mm} \mathrm{Hg}$ between upper and lower limbs (Table I).

\section{Transthoracic echocardiography and carotid} intima-media thickness

We revealed that $\mathrm{CoA}$ patients in comparison to healthy controls have higher echocardiographic parameters: left ventricular mass index (LVMI) $118.09 \pm 33.28$ vs. $96.41 \pm 19.99 \mathrm{~g} / \mathrm{m}^{2}, p<0.001$, left ventricle diastolic wall thickness: interventricular septum diameter (IVSD) 10.5 \pm 1.95 vs. $8.9 \pm 1.06 \mathrm{~mm}$ and posterior wall diameter (PWD) $9.81 \pm 1.58$ vs. $8.80 \pm 0.96 \mathrm{~mm}, p<0.001$ respectively, ascending aorta diameter $31.50 \pm 6.16$ vs. $28.03 \pm 1.79 \mathrm{~mm}$, $p<0.001$ and impaired diastolic function of the left ventricle: $E^{\prime}$ velocity $10.05 \pm 2.66$ vs. $11.33 \pm 2.61 \mathrm{~cm} / \mathrm{s}$, $p=0.034$, E/E' $10.22 \pm 5.25$ vs. $8.01 \pm 1.76, p=0.028$ and mitral annular plane systolic excursion (MAPSE) 15.03 \pm 1.65 vs. $15.90 \pm 1.03 \mathrm{~mm}, p=0.011$. Intima-media thickness of the carotid arteries was higher in the CoA group, $0.69 \pm 0.18$ vs. $0.57 \pm 0.08 \mathrm{~mm}, p<0.001$ (Table II).

We found no statistically significant differences of the left ventricle dimensions and systolic function between CoA patients and healthy controls.

When comparing echocardiographic parameters of the COA cohort with arterial hypertension (28/58) to normotensive CoA subjects (30/58) then in hypertensive CoA patients these parameters were higher: LVMI 129.84 \pm 36.79 vs. $107.13 \pm 25.68 \mathrm{~g} / \mathrm{m}^{2}, p=0.008$, IVSD $11.46 \pm 1.99$ vs. $9.60 \pm 1.45 \mathrm{~mm}, p<0.001$, PWD $10.42 \pm 1.79$ vs. 9.23 $\pm 1.10 \mathrm{~mm}, p=0.003$, CIMT $0.77 \pm 0.18$ vs. $0.61 \pm 0.14 \mathrm{~mm}$, $p<0.001$ and the left ventricle diastolic function was impaired in the hypertensive group, $E^{\prime}$ mean $9.32 \pm 3.09$ vs. $10.73 \pm 2.01 \mathrm{~cm} / \mathrm{s}, p=0.042$, E/E' $11.53 \pm 6.91$ vs. 8.99 
Table I. Baseline characteristics of study group

\begin{tabular}{|c|c|c|c|}
\hline Parameter & $\begin{array}{c}\text { CoA }(n=58) \\
22 F / 36 M\end{array}$ & $\begin{array}{c}\text { CG }(n=30) \\
12 \mathrm{~F} / 18 \mathrm{M}\end{array}$ & $P$-value \\
\hline Age [years] & $27.46 \pm 10.57$ & $27.50 \pm 8.60$ & 0.930 \\
\hline Height [cm] & $172.05 \pm 10.81$ & $174.07 \pm 8.91$ & 0.383 \\
\hline Weight [kg] & $73.21 \pm 15.06$ & $73.30 \pm 14.14$ & 0.978 \\
\hline $\mathrm{BMI}\left[\mathrm{kg} / \mathrm{m}^{2}\right]$ & $23.63 \pm 12.81$ & $24.49 \pm 9.90$ & 0.972 \\
\hline $\mathrm{BSA}\left[\mathrm{m}^{2}\right]$ & $1.84 \pm 0.24$ & $1.87 \pm 0.21$ & 0.562 \\
\hline Age at operation [years] & $8.68 \pm 8.64$ & N/D & $\mathrm{N} / \mathrm{D}$ \\
\hline Follow-up [years] & $20.39 \pm 9.8$ & N/D & $\mathrm{N} / \mathrm{D}$ \\
\hline Moderate MR (\%) & 6.89 & 0 & $\mathrm{~N} / \mathrm{D}$ \\
\hline Severe AS or AR (\%) & 0 & 0 & N/D \\
\hline Moderate AS (\%) & 12.06 & 0 & $N / D$ \\
\hline Moderate AR (\%) & 24.14 & 0 & $N / D$ \\
\hline Bicuspid aortic valve (\%) & 62.06 & 0 & $N / D$ \\
\hline Aortic valve replacement (\%) & 1.72 & 0 & N/D \\
\hline Aortic aneurysm (\%) & 0 & 0 & N/D \\
\hline Aortic dilatation (\%) & 13.79 & 0 & N/D \\
\hline Bentall de Bono surgery (\%) & 6.89 & 0 & N/D \\
\hline Hypoplastic aortic arch (\%) & 17.24 & 0 & $N / D$ \\
\hline Recoarctation (\%) & 25.86 & 0 & N/D \\
\hline Arterial hypertension (\%) & 48.31 & 0 & $N / D$ \\
\hline Coronary artery intervention (\%) & 6.89 & 0 & $N / D$ \\
\hline Cerebral vascular insult (\%) & 0 & 0 & $\mathrm{~N} / \mathrm{D}$ \\
\hline$\beta$-blocker therapy (\%) & 53.4 & 0 & $\mathrm{~N} / \mathrm{D}$ \\
\hline ACE inhibitor therapy (\%) & 36.2 & 0 & N/D \\
\hline AT1 blockers (\%) & 6.9 & 0 & $N / D$ \\
\hline Ca blockers (\%) & 17.2 & 0 & N/D \\
\hline Diuretics therapy (\%) & 22.4 & 0 & N/D \\
\hline Statin therapy (\%) & 34.2 & 0 & $\mathrm{~N} / \mathrm{D}$ \\
\hline ASA (\%) & 6.9 & 0 & N/D \\
\hline
\end{tabular}

$A R$ - aortic valve, regurgitation, $A S$ - aortic valve stenosis, $A S A$ - acetylsalicylic acid, BMI-body mass index, BSA-body surface area, $M R$ - mitral valve regurgitation. Data are presented as means with standard deviation (mean \pm SD) or as percentage (\%) of total number; $p$-value $\leq 0.05$ is considered as statistically significant.

$\pm 2.57, p=0.065$, MAPSE $15.00 \pm 2.03$ vs. $15.06 \pm 1.23 \mathrm{~mm}$, $p=0.879$.

No statistically significant differences in the echocardiography were observed between CoA subjects who underwent treatment of re-stenosis (15/58) and the cohort without residual coarctation (43/58): LVMI 110.72 \pm 33.41 vs. $120.67 \pm 33.24 \mathrm{~g} / \mathrm{m}^{2}, p=0.323$, IVSD 10.66 \pm 2.44 vs. $10.44 \pm 1.79 \mathrm{~mm}, p=0.705$, PWD $9.73 \pm 1.10$ vs. $9.83 \pm 1.73, p=0.829$, E' mean $11.13 \pm 2.29$ vs. 9.67 $\pm 2.70 \mathrm{~cm} / \mathrm{s}, p=0.067$, E/E' $8.53 \pm 2.95$ vs. $10.80 \pm 5.76$, $p=0.152$, CIMT $0.71 \pm 0.16$ vs. $0.68 \pm 0.19 \mathrm{~mm}, p=0.561$.

\section{Cardiopulmonary exercise test}

The results of the cardiopulmonary exercise tests of CoA patients in comparison with control group revealed statistically significant differences $(p<0.001)$ : $\mathrm{VO}_{2} / \mathrm{kg}$ AT and $\mathrm{VO}_{2} / \mathrm{kg}$ peak were significantly lower $20.09 \pm 6.0$ vs. $36.31 \pm 10.68$ and $29.01 \pm 8.79$ vs. $49.16 \pm 7.38 \mathrm{ml} / \mathrm{kg} /$ $\min , p<0.001$ respectively and VE/VCO 2 peak was higher, $28.18 \pm 4.69$ vs. $26.78 \pm 3.13, p=0.017$, in CoA patients than in the control group. The peak heart rate (HR) and the percentage of maximal heart rate were significantly reduced in the study group. The peak systolic blood pres- 
Table II. Echocardiographic parameters: comparison between coarctation of aorta patients and controls

\begin{tabular}{lccc}
\hline Parameter & Study group $(n=\mathbf{5 8})$ & Controls $(n=30)$ & $P$-value \\
\hline LVD $[\mathrm{m}]$ & $50.41 \pm 6.19$ & $48.66 \pm 4.75$ & 0.180 \\
\hline LVS $[\mathrm{mm}]$ & $31.26 \pm 5.17$ & $30.13 \pm 4.18$ & 0.306 \\
\hline LV EF $(\%)$ & $66.46 \pm 6.42$ & $67.16 \pm 4.76$ & 0.599 \\
\hline LVMI $\left[\mathrm{g} / \mathrm{m}^{2}\right]$ & $118.09 \pm 33.28$ & $96.41 \pm 19.99$ & 0.0015 \\
\hline IVSD $[\mathrm{mm}]$ & $10.50 \pm 1.96$ & $8.90 \pm 1.06$ & $<0.001$ \\
\hline PWD $[\mathrm{mm}]$ & $9.81 \pm 1.58$ & $8.80 \pm 0.96$ & 0.0019 \\
\hline Ascending aorta $[\mathrm{mm}]$ & $31.50 \pm 6.16$ & $28.03 \pm 1.79$ & 0.003 \\
\hline Maximal gradient at coarctation $[\mathrm{mm} \mathrm{Hg}]$ & $25.86 \pm 12.96$ & $6.65 \pm 1.56$ & $<0.001$ \\
\hline E' mean [cm/s] & $10.05 \pm 2.66$ & $11.33 \pm 2.61$ & 0.034 \\
\hline E/E' & $10.21 \pm 5.25$ & $8.01 \pm 1.76$ & 0.028 \\
\hline MAPSE $[\mathrm{mm}]$ & $15.03 \pm 1.65$ & $15.90 \pm 1.03$ & 0.011 \\
\hline CIMT $[\mathrm{mm}]$ & $0.69 \pm 0.18$ & $0.57 \pm 0.08$ & $<0.001$
\end{tabular}

CIMT - carotid intima-media thickness, IVSD - interventricular septum diameter in diastole, LV EF-left ventricle ejection fraction, LVD - left ventricle diastolic dimension, LVMI - left ventricle mass index, LVS - left ventricle systolic dimension, MAPSE - mitral annular plane systolic excursion, PWD - posterior wall diameter in diastole. Data are presented as means with standard deviation (mean \pm SD) or as percentage (\%) of total number; pvalue $\leq 0.05$ is considered as statistically significant.

sure was higher in the CoA cohort when exercise capacity as metabolic equivalents (METs) and duration of exercise were lower. Significant correlations between VE/VCO 2 and duration of CPET the test $(r=0.35, p=0.007)$, HR peak $(r=0.29, p=0.022)$, peak workload $(r=0.51, p<0.001)$ and peak blood pressure were found $(r=0.29, p=0.023)$. In the whole CoA group there were no significant correla- tions between CPET parameters and age, BMI or time from surgery (Table III).

In comparison between hypertensive CoA patients (28/58) and normotensive CoA patients (30/58), heart rate peak was lower, $149.75 \pm 20.77$ vs. $164.30 \pm 21.52$ bpm, $p=0.011$, and systolic blood pressure peak higher in the hypertensive population, $182.93 \pm 14.70$ vs. 167.20

Table III. Cardiopulmonary exercise test: comparison of coarctation of aorta patients to control group

\begin{tabular}{|c|c|c|c|}
\hline Parameter & Study group $(n=58)$ & Controls $(n=30)$ & $P$-value \\
\hline SBP resting [mm Hg] & $136.55 \pm 16.27$ & $123.47 \pm 10.34$ & $<0.001$ \\
\hline DBP resting [mm Hg] & $74.95 \pm 12.29$ & $74.83 \pm 6.91$ & 0.955 \\
\hline$\%$ AT predicted & $65.07 \pm 18.34$ & $94.57 \pm 2.79$ & $<0.001$ \\
\hline Time peak [min] & $15.28 \pm 3.21$ & $16.82 \pm 2.78$ & 0.027 \\
\hline HR peak [bpm] & $157.28 \pm 22.22$ & $177.93 \pm 23.08$ & $<0.001$ \\
\hline$\%$ HR max & $78.81 \pm 14.72$ & $95.17 \pm 2.96$ & $<0.001$ \\
\hline $\mathrm{VO}_{2} / \mathrm{kg}$ peak $[\mathrm{ml} / \mathrm{kg} / \mathrm{min}]$ & $29.01 \pm 8.79$ & $49.16 \pm 7.38$ & $<0.001$ \\
\hline$\% \mathrm{VO}_{2} / \mathrm{kg}$ predicted & $81.91 \pm 20.81$ & $95.97 \pm 3.92$ & $<0.001$ \\
\hline RER peak & $1.11 \pm 0.08$ & $1.12 \pm 0.09$ & 0.415 \\
\hline VE/VCO ${ }_{2}$ peak & $28.18 \pm 4.69$ & $26.78 \pm 3.13$ & 0.017 \\
\hline BR peak & $45.73 \pm 23.11$ & $41.76 \pm 16.95$ & 0.414 \\
\hline MET peak & $11.03 \pm 2.72$ & $12.49 \pm 0.71$ & 0.005 \\
\hline SBP peak $[\mathrm{mm} \mathrm{Hg}]$ & $174.79 \pm 17.62$ & $153.33 \pm 4.79$ & $<0.001$ \\
\hline DBP peak [mm Hg] & $77.00 \pm 14.91$ & $80.00 \pm 0.21$ & 0.275 \\
\hline
\end{tabular}

BR peak - peak breathing reserve, DBP-diastolic blood pressure, HR peak - heart rate peak, \% HR max - percentage of maximal heart rate, SBP-systolic blood pressure, RER peak - peak respiratory exchange ratio, $V \mathrm{CO}_{2}$ peak - peak carbon dioxide production, $V \mathrm{VO}_{2}$ rest - carbon dioxide production at rest, VEIVCO, peak - peak ventilatory equivalent for $\mathrm{CO}_{2}, \mathrm{VO}_{2} / \mathrm{kg} A T$ - oxygen uptake per $\mathrm{kg}$ at anaerobic threshold, $\mathrm{VO}_{2} / \mathrm{kg}$ peak - peak oxygen uptake per $\mathrm{kg}, \mathrm{VO}_{2} / \mathrm{kg}$ rest - oxygen uptake per $\mathrm{kg}$ at rest, $\mathrm{VO}_{2}$ peak - peak oxygen uptake, $\mathrm{VO}_{2}$ rest - oxygen uptake at rest. Data are presented as means with standard deviation (mean \pm SD) or as percentage (\%) of total number; $p$-value $\leq 0.05$ is considered as statistically significant. 
$\pm 16.89 \mathrm{~mm} \mathrm{Hg}, p<0.001$. The $\mathrm{VO}_{2} / \mathrm{kg}$ peak and the percentage of predicted value for age were similar in these groups: $29.27 \pm 9.90$ vs. $28.76 \pm 7.77 \mathrm{ml} / \mathrm{kg} / \mathrm{min}, p=0.828$, $83.86 \pm 22.19$ vs. $80.10 \pm 19.64 \%, p=0.497$ respectively. There was no statistically significant difference between the groups in $\mathrm{VE} / \mathrm{VCO}_{2}$ peak: $24.50 \pm 3.17$ vs. $26.08 \pm 4.36$ $\mathrm{ml} / \mathrm{kg} / \mathrm{min}, p=0.120$, duration of the test: $15.24 \pm 2.99$ vs. $15.30 \pm 3.44 \mathrm{~min}, p=0.948$ and maximal workload: $11.24 \pm 2.49$ vs. $10.83 \pm 2.94$ MET, $p=0.562$ (Table IV).

No statistically significant differences in the cardiopulmonary results were observed between CoA subjects who underwent treatment of re-stenosis (15/58) and the cohort without residual coarctation (43/58): $\mathrm{VO}_{2} / \mathrm{kg}$ peak $28.13 \pm 8.77$ vs. $29.31 \pm 8.87 \mathrm{ml} / \mathrm{kg} / \mathrm{min}, p=0.656$, $\mathrm{VE} / \mathrm{VCO}_{2}$ peak $24.31 \pm 3.20$ vs. $25.67 \pm 4.07 \mathrm{ml} / \mathrm{kg} / \mathrm{min}$, $p=0.243$, maximal workload $11.16 \pm 2.55$ vs. 10.98 $\pm 2.80 \mathrm{MET}, p=0.822$, SBP peak $180.33 \pm 16.95$ vs. 172.86 $\pm 17.62 \mathrm{~mm} \mathrm{Hg}, p=0.159$, DBP peak $81.33 \pm 15.52$ vs. $75.49 \pm 14.57 \mathrm{~mm} \mathrm{Hg}, p=0.194$ (Table V).

\section{4-hour ambulatory blood pressure measurement}

The results of the 24-hour ABPM of CoA patients in comparison with the control group revealed statistically significant differences: daytime systolic blood pressure (SBP) and diastolic blood pressure (DBP) were higher, $128.13 \pm 12.47$ vs. $106.43 \pm 6.23 \mathrm{~mm} \mathrm{Hg}$ and $74.62 \pm 9.60$ vs. $69.83 \pm 5.29 \mathrm{~mm} \mathrm{Hg}, p<0.001$ and $p=0.013$ respectively; the night values of SBP and DBP were also significantly raised in the CoA cohort (112.08 \pm 13.63 vs. 97.36 $\pm 10.41 \mathrm{~mm} \mathrm{Hg}$ and $62.91 \pm 9.17$ vs. $59.03 \pm 8.28 \mathrm{~mm} \mathrm{Hg}$, $p<0.001$ and $p=0.026$ respectively.

When analysing ABPM results in the hypertensive CoA cohort in comparison to the normotensive CoA cohort, the SBP during the day and the night time were higher in the first group, $134.46 \pm 10.36$ vs. $122.23 \pm 11.45$ $\mathrm{mm} \mathrm{Hg}$ and $118.60 \pm 12.28$ vs. $106.03 \pm 12.06 \mathrm{~mm} \mathrm{Hg}$, $p<0.001$; no other statistically significant differences between these groups were exposed in ABPM.

In comparison of ABPM measurements in COA subjects who underwent treatment of re-stenosis $(15 / 58)$ and the cohort without residual coarctation (43/58) there were no statistically significant differences in SBP and DBP values during the day and night time between the groups.

In the whole CoA group, we revealed a statistically significant correlation of systolic blood pressure in ABPM with: left ventricle mass index $(r=0.29, p=0.025)$ and

Table IV. Comparison of hypertensive to normotensive coarctation of aorta patients

\begin{tabular}{|c|c|c|c|}
\hline Parameter & $\mathrm{H} \mathrm{CoA}(n=28)$ & N CG $(n=30)$ & $P$-value \\
\hline Age [years] & $33.50 \pm 12.23$ & $25.73 \pm 7.12$ & 0.004 \\
\hline Age at operation [years] & $10.57 \pm 14.89$ & $6.76 \pm 9.34$ & 0.245 \\
\hline Follow-up [years] & $22.07 \pm 11.56$ & $18.96 \pm 7.78$ & 0.232 \\
\hline Hypoplastic aortic arch (\%) & 28.57 & 6.66 & 0.027 \\
\hline Recoarctation (\%) & 39.28 & 13.33 & 0.024 \\
\hline HR peak [bpm] & $149.75 \pm 20.77$ & $164.30 \pm 21.52$ & 0.011 \\
\hline$\%$ HR max & $75.21 \pm 13.50$ & $82.17 \pm 15.22$ & 0.071 \\
\hline $\mathrm{VO}_{2} / \mathrm{kg}$ peak $[\mathrm{ml} / \mathrm{kg} / \mathrm{min}]$ & $29.27 \pm 9.90$ & $28.76 \pm 7.77$ & 0.828 \\
\hline$\% \mathrm{VO}_{2} / \mathrm{kg}$ peak predicted & $83.86 \pm 22.19$ & $80.10 \pm 19.64$ & 0.497 \\
\hline VE/VCO ${ }_{2}$ peak & $24.50 \pm 3.17$ & $26.08 \pm 4.36$ & 0.120 \\
\hline SBP peak [mm Hg] & $182.93 \pm 14.70$ & $167.20 \pm 16.89$ & $<0.001$ \\
\hline DBP peak [mm Hg] & $80.82 \pm 15.19$ & $73.43 \pm 13.96$ & 0.058 \\
\hline LVMI $\left[\mathrm{g} / \mathrm{m}^{2}\right]$ & $129.84 \pm 36.79$ & $107.13 \pm 25.68$ & 0.008 \\
\hline IVSD [mm] & $11.46 \pm 1.99$ & $9.60 \pm 1.45$ & $<0.001$ \\
\hline PWD $[\mathrm{mm}]$ & $10.42 \pm 1.79$ & $9.23 \pm 1.10$ & 0.003 \\
\hline$E^{\prime}$ mean $[\mathrm{cm} / \mathrm{s}]$ & $9.32 \pm 3.09$ & $10.73 \pm 2.01$ & 0.042 \\
\hline$E / E^{\prime}$ & $11.53 \pm 6.91$ & $8.99 \pm 2.57$ & 0.065 \\
\hline CIMT $[\mathrm{mm}]$ & $0.77 \pm 0.18$ & $0.61 \pm 0.14$ & $<0.001$ \\
\hline
\end{tabular}

CIMT - carotid intima-media thickness, DBP-diastolic blood pressure, HR peak - heart rate peak, \% HR max - percentage of maximal heart rate, IVSD - interventricular septum diameter in diastole, LVMI - left ventricle mass index, PWD - posterior wall diameter in diastole, SBP-systolic blood pressure, VENVCO 2 peak - peak ventilatory equivalent for $\mathrm{CO}_{2}, \mathrm{VO} / \mathrm{kg}$ peak - peak oxygen uptake per $\mathrm{kg}, \mathrm{VO} / \mathrm{kg}$ rest - oxygen uptake per $\mathrm{kg}$ at rest, $\mathrm{VO}_{2}$ peak - peak oxygen uptake. Data are presented as means with standard deviation (mean $+S D)$ or as percentage (\%) of total number; $p$-value $\leq 0.05$ is considered as statistically significant. 
Table V. Comparison of recoarctation to no-recoarctation of aorta patients

\begin{tabular}{|c|c|c|c|}
\hline Parameter & $\operatorname{Re} \operatorname{CoA}(n=15)$ & NoRe CoA $(n=43)$ & $P$-value \\
\hline Age [years] & $26.26 \pm 6.34$ & $29.93 \pm 11.12$ & 0.233 \\
\hline Age at operation [years] & $5.80 \pm 5.87$ & $9.58 \pm 13.87$ & 0.312 \\
\hline Follow-up [years] & $20.73 \pm 9.41$ & $20.37 \pm 10.07$ & 0.903 \\
\hline Hypoplastic aortic arch (\%) & 46.66 & 6.97 & $<0.001$ \\
\hline Arterial hypertension (\%) & 73.33 & 39.53 & $<0.001$ \\
\hline HR peak [bpm] & $62.93 \pm 15.25$ & $65.81 \pm 19.40$ & 0.604 \\
\hline$\%$ HR max & $71.93 \pm 14.51$ & $81.21 \pm 14.17$ & 0.034 \\
\hline $\mathrm{VO}_{2} / \mathrm{kg}$ peak $[\mathrm{ml} / \mathrm{kg} / \mathrm{min}]$ & $28.13 \pm 8.77$ & $29.31 \pm 8.87$ & 0.656 \\
\hline$\% \mathrm{VO}_{2} / \mathrm{kg}$ peak predicted & $77.47 \pm 19.85$ & $83.46 \pm 21.14$ & 0.341 \\
\hline $\mathrm{VE} / \mathrm{VCO}_{2}$ peak & $24.31 \pm 3.20$ & $25.67 \pm 4.07$ & 0.243 \\
\hline SBP peak $[\mathrm{mm} \mathrm{Hg}]$ & $180.33 \pm 16.95$ & $172.86 \pm 17.62$ & 0.159 \\
\hline DBP peak [mm Hg] & $81.33 \pm 15.52$ & $75.49 \pm 14.57$ & 0.194 \\
\hline LVMI $\left[\mathrm{g} / \mathrm{m}^{2}\right]$ & $110.72 \pm 33.41$ & $120.67 \pm 33.24$ & 0.323 \\
\hline IVSD [mm] & $10.66 \pm 2.44$ & $10.44 \pm 1.79$ & 0.705 \\
\hline $\mathrm{PWD}[\mathrm{mm}]$ & $9.73 \pm 1.10$ & $9.83 \pm 1.73$ & 0.829 \\
\hline$E^{\prime}$ mean $[\mathrm{cm} / \mathrm{s}]$ & $11.13 \pm 2.29$ & $9.67 \pm 2.70$ & 0.067 \\
\hline$E / E^{\prime}$ & $8.53 \pm 2.95$ & $10.80 \pm 5.76$ & 0.152 \\
\hline CIMT [mm] & $0.71 \pm 0.16$ & $0.68 \pm 0.19$ & 0.561 \\
\hline
\end{tabular}

CIMT - carotid intima-media thickness, DBP - diastolic blood pressure, HR peak - heart rate peak, \% HR max - percentage of maximal heart rate, IVSD - interventricular septum diameter in diastole, LVMI - left ventricle mass index, PWD - posterior wall diameter in diastole, SBP-systolic blood pressure, VENVCO peak - peak ventilatory equivalent for $\mathrm{CO}_{2}, \mathrm{VO}_{2} / \mathrm{kg}$ peak - peak oxygen uptake per $\mathrm{kg}, \mathrm{VO} / \mathrm{kg}$ rest - oxygen uptake per $\mathrm{kg}$ at rest, VO $\mathrm{O}_{2}$ peak - peak oxygen uptake. Data are presented as means with standard deviation (mean \pm SD) or as percentage (\%) of total number; $p$-value $\leq 0.05$ is considered as statistically significant.

wall thickness $(r=0.31, p=0.039)$. Older age at operation was related to left ventricle walls thickness $(r=0.27$, $p=0.041)$ and carotid intima-media thickness $(r=0.26$, $p=0.046$ ). Statistical analysis showed no association of any cardio-pulmonary parameters with time from surgery, type of operation or echocardiography results.

\section{Discussion}

The principal finding in the present study is that in patients after aortic coarctation repair despite successful short-term outcomes late cardiovascular complications occur.

The main comorbidity after successful repair of aortic coarctation is arterial hypertension, present in $48.3 \%$ of subjects in our study, which is compatible with literature data, 32.5\% (range: 25-68\%) [11]. Hypertensive patients in our study were older. Many of them were treated due to recoarctation and suffered from hypoplastic aortic arch. Patients with arterial hypertension presented higher mass of the left ventricle, greater wall thickness and greater carotid intima-media thickness. They had impaired left ventricle diastolic function. These changes are common in patients with arterial hypertension and widely described in the literature [17-20]. Causes of arterial hypertension in coarctation of aorta patients are multifactorial. The aorta wall, in the section before the narrowing, has a different structure, contains more collagen and less elastin fibre, and lacks smooth muscle cells. The aortic wall stiffness is increased [9], sensitivity reduced and the aorta mechanoreceptors activated. The pathophysiological processes are initiated with an advantage of the sympathetic system, changes in the endocrine system, endothelial dysfunction and remodelling of the vessels, which leads to increase of the peripheral vascular resistance and increase in blood pressure [20]. Data from the literature [21] indicate that among congenital heart diseases arterial hypertension is the most frequent in coarctation of the aorta. The discussion about conditions of coarctation repair (time of intervention and type of operation) to avoid arterial hypertension is still open. In our study patients with arterial hypertension were older and operated on at older age than normotensive subjects. The observations from other studies [22] reveal that younger age at operation is related to lower arterial pressure at follow-up; it is the best if treatment is applied before $9-10$ years old. We did not observe the 
influence of type of surgery on development of arterial hypertension. The opposite conclusion was presented by Giordano et al. [23] - patients after subclavian flap surgery in comparison to end-to-end anastomosis showed a higher incidence of hypertension. The authors explain that fact by greater resection of the abnormal aortic tissue in the second method and lower aortic stiffness as a consequence. Our observations from cardiopulmonary exercise tests revealed that raised initial arterial pressure in the exercise test is a marker of hypertensive disease in CoA subjects. All hypertensive patients presented higher values of the resting arterial pressure in CPET than normotensive ones. On the other hand, only 5 out of 58 patients (8.62\%) reached values above $200 / 110 \mathrm{~mm} \mathrm{Hg}$ at peak workload, which was considered as a hypertensive reaction to exercise test. All of them were treated due to arterial hypertension before. Many authors [24-30] have analysed arterial pressure reaction on effort during the exercise tests. The main conclusions are that elevated arterial pressure at the beginning of the test and hypertensive reaction at peak exercise are the symptoms of arterial hypertension in longer follow-up.

In the present research, the cardiopulmonary exercise tests of CoA patients in comparison to controls revealed lower values of: $\mathrm{VO}_{2} / \mathrm{kg}$ peak, heart rate peak, \% max $H R$, exercise capacity as metabolic equivalents and duration of an exercise. The reason for these results is still uncertain. There may be some influence of chronotropic incompetence, which occurred in $27.58 \%$ of subjects if described as maximal heart rate percent below $80 \%$ at peak workload. $\beta$-blocker therapy was suspended $48 \mathrm{~h}$ before to avoid its impact on heart rate. Data from the literature [31, 32] indicate that chronotropic incompetence in adults after a congenital heart disease operation was related to lower $\mathrm{VO}_{2} / \mathrm{kg}$ peak and higher risk of cardiac insufficiency in future. We revealed significant correlations between $\mathrm{VE} / \mathrm{VCO}_{2}$ and duration of CPET, HR peak, peak workload (METs) and peak blood pressure. No other associations were found.

In our study all patients with restenosis (25.86\%) underwent cardiac catheterization before this research and were treated with percutaneous angioplasty or stent implantation with a successful result in hemodynamic measurement. Most of them, despite the effective intervention, suffered from arterial hypertension. Data from the literature [33-40] demonstrate a decrease of arterial pressure after percutaneous intervention, although still higher values than in the healthy population. In our observation, patients with aortic recoarctation had more often concomitant hypoplastic aortic arch. Age at operation and length of follow-up had no impact on aortic restenosis occurrence. We did not observe statistically significant differences between any of the echocardiography or cardiopulmonary test results between patients with recoarctation and the no-recoarctation ones. In case of recurrent significant coarctation of the aorta, invasive treatment is recommended as soon as possible, with modification of hypotensive therapy if needed [41].

A significant comorbidity among CoA patients is aortic aneurysm formation. This process is related to disorders of thoracic aorta wall structure and impaired mechanical function of aortic tissue. Arterial hypertension, which is common in this population, additionally has a positive impact on rise in tension of the aortic walls. More frequent aortic aneurysm development is observed in patients with coexisting bicuspid aortic valve, which can be explained by a general vascular disorder of the thoracic aorta in both groups. In our research we did not observe aortic aneurysm of any patients, but $6.89 \%$ of our CoA patients had undergone a Bentall de Bono operation. Furthermore, ascending aorta diameter was higher in the CoA group than in controls and $13.79 \%$ of CoA subjects had ascending aorta dilatation. All the cases were related to presence of bicuspid aortic valve. We did not reveal any association between ascending aorta diameter and type of aortic coarctation surgery. Data from the literature [42-44] show that aneurysms are more common after patch aortoplasty technique and transcatheter interventions, particularly balloon angioplasty without stent implantation.

A significant comorbidity after successful aortic coarctation repair is premature atherosclerosis. In our observations $6.89 \%$ of patients had confirmed coronary artery disease in angiography. These patients were older than the others from the CoA group, were operated on due to CoA in older age (after 18 years old) and suffered from concomitant cardio-vascular risk factors (e.g. hyperlipidemia, obesity and diabetes mellitus). Other authors have reported $[7,45]$ that coarctation of aorta does not increase risk of coronary artery disease itself, but due to concomitant arterial hypertension and endothelial dysfunction. Strict control of conventional atherosclerosis risk factors after coarctation repair reduces long-tem vascular risk.

\section{Study limitations}

Several limitations of the study should be acknowledged. First, the number of patients in the study was small. It is representative for a patient population in real-life clinical practice, but a larger study group is expected. Second, the hypertensive patients in our study were under treatment such as ACE inhibitors and $\beta$-blockers, drugs that have an influence on cardio-pulmonary test results. The therapy was suspended $48 \mathrm{~h}$ before the examination to reduce its impact on measurement results. Certainly, longer follow-up is required; some comorbidities may occur at a later time.

\section{Conclusions}

Despite successful aortic coarctation repair and positive short-term outcomes, adult patients in longer fol- 
low-up are exposed to arterial hypertension, vascular complications such as recurrent aortic stenosis, aneurysms formation and premature atherosclerosis. They have reduced exercise capacity, which is related to hypertensive reaction, increased arterial stiffness and chronotropic incompetence. Exercise intolerance occurs as a result of lowered oxygen uptake and increased ventilatory response. These patients require regular follow-up to reduce long-term morbidity and mortality after coarctation repair.

\section{Conflict of interest}

The authors declare no conflict of interest.

\section{References}

1. Vonder Muhll IF, Sehgal T, Paterson DI. The adult with repaired coarctation: need for lifelong surveillance. Can J Cardiol 2016; 32: 1038.e11-5.

2. Usman MH, Rengifo-Moreno P, Janzer SF, et al. Coarctation of the aorta: management, indications for intervention, and advances in care. Curr Treat Options Cardiovasc Med 2014; 16: 341.

3. Brown ML, Burkhart HM, Connolly HM, et al. Coarctation of the aorta: lifelong surveillance is mandatory following surgical repair. J Am Coll Cardiol 2013; 62: 1020-5.

4. Torok RD, Campbell MJ, Fleming GA, et al. Coarctation of the aorta: management from infancy to adulthood. World J Cardiol 2015; 7: 765-75.

5. Toro-Salazar O, Steinberger J, Thomas W, et al. Long-term follow-up of patients after coarctation of the aorta repair. Am J Cardiol 2002; 89: 541-7.

6. Tanous D, Benson L, Horlick E. Coarctation of the aorta: evaluation and management. Curr Opin Cardiol 2009; 24: 509-15.

7. Cook S, Ferketich A, Raman S. Myocardial ischemia in asymptomatic adults with repaired aortic coarctation. Int J Cardiol 2009; 133: 95-101.

8. Swan L, Kraidly M, Vonder Muhll I, et al. Surveillance of cardiovascular risk in the normotensive patient with repaired aortic coarctation. Int J Cardiol 2010; 139: 283-8.

9. Róg B, Okólska M, Dziedzic-Oleksy $\mathrm{H}$, et al. Arterial stiffness in adult patients after coarctation of aorta repair and with bicuspid aortic valve. Acta Cardiol doi: 10.1080/00015385.2018.1530084.

10. Róg B, Okólska M, Sałapa K, et al. Cardiovascular risk factors in patients after coarctation of aorta repair. Pol Merkur Lekarski 2018; 44: 219-22.

11. Canniffe C, Ou P, Walsh K, et al. Hypertension after repair of aortic coarctation: a systematic review. Int J Cardiol 2013; 167: 2456-61.

12. Roifman I, Therrien J, Ionescu-Ittu R, et al. Coarctation of the aorta and coronary artery disease: fact or fiction? Circulation 2012; 126: 16-21.

13. Williams B, Mancia G, Spiering W, et al. 2018 ESC/ESH Guidelines for the management of arterial hypertension. Kardiol Pol 2019; 77: 71-159.

14. Ravani A, Werba JP, Frigerio B, et al. Assessment and relevance of carotid intima-media thickness (C-IMT) in primary and secondary cardiovascular prevention. Curr Pharm Des 2015; 21: 1164-71.

15. Tomkiewicz-Pajak L, Podolec P, Drabik L, et al. Single ventricle function and exercise tolerance in adult patients after Fontan operation. Acta Cardiol 2014; 69: 155-60.
16. Takken T, Blank AC, Hulzebos EH, et al. Cardiopulmonary exercise testing in congenital heart diseases: (contra) indication and interpretation. Neth Heart J 2009; 10: 385-92.

17. Swan L, Goyal S, Hsia C, et al. Exercise systolic blood pressures are of questionable value in the assessment of the adult with a previous coarctation repair. Heart 2003; 89: 189-92.

18. de Divitiis M, Pilla C, Kattenhorn M, et al. Ambulatory blood pressure, left ventricular mass, and conduit artery function late after successful repair of coarctation of the aorta. J Am Coll Cardiol 2003; 41: 2259-65.

19. Crepaz R, Pitscheider W, Oberhollenzer R, et al. Long-term follow-up in patients operated on for aortic coarctation. The echo-Doppler and MRI assessment of left ventricular function and the transisthmic gradient. G Ital Cardiol 1993; 23: 767-76.

20. O'Sullivan J. Late hypertension in patients with repaired aortic coarctation. Curr Hypertens Rep 2014; 16: 421.

21. Roche S, Silversides C. Hypertension, obesity, and coronary artery disease in the survivors of congenital heart disease. Can J Cardiol 2013; 29: 841-8.

22. Lillitos PJ, Nassar MS, Tibby S, et al. Is the medical treatment for arterial hypertension after primary aortic coarctation repair related to age at surgery? A retrospective cohort study. Cardiol Young 2017; 27: 1701-7.

23. Giordano U, Giannico S, Turchetta A, et al. The influence of different surgical procedures on hypertension after repair of coarctation. Cardiol Young 2005; 15: 477-80.

24. Das B, Raj S, Shoemaker L. Exercise testing is useful to screen for residual coarctation in children. Pediatr Cardiol 2009; 30: 763-7.

25. Luijendijk P, Bouma BJ, Vriend JW, et al. Usefulness of exercise-induced hypertension as predictor of chronic hypertension in adults after operative therapy for aortic isthmic coarctation in childhood. Am J Cardiol 2011; 108: 435-9.

26. Markham LW, Knecht SK, Daniels SR, et al. Development of exercise-induced arm-leg blood pressure gradient and abnormal arterial compliance in patients with repaired coarctation of the aorta. Am J Cardiol 2004; 94: 1200-2.

27. Canniffe C, Ou P, Walsh K, et al. Hypertension after repair of aortic coarctation: a systematic review. Int J Cardiol 2013; 167 : 2456-61.

28. Madueme PC, Khoury PR, Urbina EM, et al. Predictors of exaggerated exercise-induced systolic blood pressures in young patients after coarctation repair. Cardiol Young 2013; 23: 416-22.

29. Correia AS, Gonçalves A, Paiva M, et al. Long-term follow-up after aortic coarctation repair: the unsolved issue of exerciseinduced hypertension. Rev Port Cardiol 2013; 32: 879-83.

30. Vriend JW, van Montfrans GA, Romkes HH, et al. Relation between exercise-induced hypertension and sustained hypertension in adult patients after successful repair of aortic coarctation. J Hypertens 2004; 22: 501-9.

31. Norozi K, Wessel A, Alpers V, et al. Chronotropic incompetence in adolescents and adults with congenital heart disease after cardiac surgery. J Card Fail 2007; 13: 263-8.

32. Róg B, Sałapa K, Okólska M, et al. Clinical evaluation of exercise capacity in adults with systemic right ventricle. Tex Heart Inst J 2019; 46: 14-20.

33. Hamid T, Motwani M, Schneider $\mathrm{H}$, et al. Benefit of endovascular stenting for aortic coarctation on systemic hypertension in adults. Arch Cardiovasc Dis 2015; 108: 626-33.

34. De Caro E, Spadoni I, Crepaz R, et al. Stenting of aortic coarctation and exercise-induced hypertension in the young. Catheter Cardiovasc Interv 2010; 75: 256-61. 
35. Weber H, Cyran S. Endovascular stenting for native coarctation of the aorta is an effective alternative to surgical intervention in older children. Congenit Heart Dis 2008; 3: 54-9.

36. Musto C, Cifarelli A, Pucci E, et al. Endovascular treatment of aortic coarctation: long-term effects on hypertension. Int J Cardiol 2008; 130: 420-5.

37. Morrical B, Anderson J, Taggart N. Exercise capacity before and after stent placement for coarctation of the aorta: a single-center case series. Pediatr Cardiol 2017; 38: 1143-7.

38. Morgan G, Lee K, Chaturvedi R, et al. Systemic blood pressure after stent management for arch coarctation implications for clinical care. JACC Cardiovasc Interv 2013; 6: 192-201.

39. Agnoletti G, Bonnet C, Bonnet D, et al. Mid-term effects of implanting stents for relief of aortic recoarctation on systemic hypertension, carotid mechanical properties, intimal medial thickness and reflection of the pulse wave. Cardiol Young 2005; 15 : 245-50.

40. Hamid T, Motwani M, Schneider H, et al. Benefit of endovascular stenting for aortic coarctation on systemic hypertension in adults. Arch Cardiovasc Dis 2015; 108: 626-33.

41. Baumgartner H, Bonhoeffer P, De Groot NM, et al. ESC Guidelines for the management of grown-up congenital heart disease (new version 2010). Eur Heart J 2010; 31: 2915-57.

42. Vonder Muhll I, Sehgal T, Paterson D. The adult with repaired coarctation: need for lifelong surveillance. Can J Cardiol 2016; 32: 1038.e11-5.

43. von Kodolitsch Y, Aydin M, Koschyk D, et al. Predictors of aneurysmal formation after surgical correction of aortic coarctation. J Am Coll Cardiol 2002; 39: 617-24.

44. Clair M, Fernandes S, Khairy P, et al. Aortic valve dysfunction and aortic dilation in adults with coarctation of the aorta. Congenit Heart Dis 2014; 9: 235-43.

45. Roifman I, Therrien J, Ionescu-Ittu R, et al. Coarctation of the aorta and coronary artery disease: fact or fiction? Circulation 2012; 126: 16-21. 